\title{
The logic and challenges of imaging sarcoidosis with whole body FDG PET
}

\author{
Roberto C. Valentin, MD, and Pradeep Bhambhvani, $M^{a}$ \\ a Division of Molecular Imaging and Therapeutics, Department of Radiology, The University of \\ Alabama at Birmingham, Birmingham, AL
}

Received Jul 31, 2017; accepted Aug 1, 2017

doi: 10.1007/s12350-017-1037-2

\section{See related article, pp. 486-492}

Sarcoidosis is an idiopathic systemic granulomatous disease that can affect multiple organs. The adjusted annual incidence among black Americans is roughly three times that among white Americans (35.5 cases per $100,000$, as compared with 10.9 per 100,000$).{ }^{1}$ Sarcoid involvement of the lungs and/or heart can lead to significant morbidity and mortality. Even though cardiac sarcoidosis (CS) appears to be underdiagnosed clinically, it is considered the second leading cause of death by sarcoidosis in the United States and the leading cause in Japan. ${ }^{2,3}$ Inflammatory granulomas or post-inflammatory scarring may lead to conduction abnormalities, arrhythmias, sudden cardiac death, and congestive heart failure. CS frequency is debated, with an approximate incidence of $5 \%$ based on clinical assessment, ${ }^{4} 27 \%$ in autopsy studies, ${ }^{5}$ and $39 \%$ in an imaging study that used cardiac MRI or PET. ${ }^{6}$ Obtaining ante-mortem diagnosis of cardiac sarcoidosis is challenging as the diagnostic gold standard, i.e., biopsy is invasive, subject to sampling error $^{7}$ and has a sensitivity of only $20 \%-30 \%$, because it misses the often patchy areas of cardiac involvement. ${ }^{1,8,9}$ Additionally, the criteria for the diagnosis of cardiac sarcoidosis from the Japanese Ministry of Health and Welfare (JMHW) have an imperfect diagnostic accuracy. ${ }^{10,11}$ Therefore, the diagnosis of cardiac sarcoidosis often relies on integrating both clinical and imaging findings, including those from advanced imaging

Reprint requests: Pradeep Bhambhvani, MD, Division of Molecular Imaging and Therapeutics, Department of Radiology, The University of Alabama at Birmingham, 619 19th Street South, JT 777, Birmingham, AL 35249; pbhambhvani@uabmc.edu

J Nucl Cardiol 2019;26:493-6.

$1071-3581 / \$ 34.00$

Copyright (C) 2017 American Society of Nuclear Cardiology. modalities like cardiac MRI with gadolinium and FDG PET (latter most often combined with CT, i.e. PET-CT). ${ }^{7}$ While the typical delayed gadolinium enhancement seen on cardiac MRI represents sarcoid inflammation related cardiac damage and scarring, FDG uptake represents active inflammation. Both FDG PET and MRI provide high sensitivity for diagnosing cardiac sarcoidosis, but the specificity of cardiac MRI is better. ${ }^{12}$ Advantages of FDG PET include the biologic nature of the imaging signal, the ability to identify cardiac and extracardiac sarcoidosis involvement in one exam, feasibility of imaging patients with electrical/metallic devices and renal impairment, assessment of therapy response and risk stratification. $7,13,14$

In the present issue of the Journal of Nuclear Cardiology, Patel and colleagues from the University of Illinois, Chicago, investigate the relationship of extrathoracic sarcoid findings in patients with cardiac sarcoidosis using FDG PET-CT, performed after a 72hour high fat, high protein, and very low carbohydrate (HFHPVLC) diet. ${ }^{15}$ Their aim was to determine if sarcoid patients being evaluated for cardiac involvement would benefit from whole body FDG PET-CT imaging (skull base to thighs) versus the limited thoracic imaging offered by cardiac MRI. A total of 191 consecutive FDG PET-CT scans were reviewed, of which 20 scans (10\%) were positive and six scans indeterminate $(3 \%)$ for CS. Of the 20 CS positive scans, 12 (60\%) were negative for extrathoracic sarcoid findings and 8 (40\%) showed extrathoracic involvement. Also, of the 165 scans (86\%) that were negative for CS, 56 (34\%) were positive for extrathoracic sarcoid involvement. Their findings make a persuasive argument in favor of whole body FDG PET-CT for diagnosis of not only cardiac sarcoidosis, but also extrathoracic sites of involvement, which cannot be assessed with the limited field of view cardiac MRI. Additionally, while MRI has the advantage of providing dual structural and functional information, its spatial resolution in the lungs is inferior to $\mathrm{CT},{ }^{16}$ which 
could be an added advantage of FDG PET-CT over cardiac MRI for characterizing pulmonary findings. While most patients with sarcoidosis have pulmonary involvement, Patel et al. did not report the incidence of sarcoid related pulmonary and other thoracic findings in their cohort.

Patient preparation plays an extremely important role in determining cardiac FDG PET image quality. Numerous preparation protocols aimed at suppressing physiologic myocardial glucose utilization/FDG uptake have been described without a clear consensus on the optimal method. A 1-2 meal high fat, high protein, low to no carbohydrate diet followed by at least 4-hours of fasting, with or without intravenous heparin $(50 \mathrm{IU} / \mathrm{kg})$ 15-minutes prior to FDG injection, have been shown to be successful in suppressing physiologic myocardial FDG uptake in $85 \%-100 \%$ of cases. ${ }^{14,17-21}$ Patel and colleagues prepared their patients with a 72-hour HFHPVLC diet protocol that included breakfast approximately 4 hours before scanning, and were able to successfully suppress background myocardial FDG uptake and minimize indeterminate findings. ${ }^{15}$ Previously their group had proven effectiveness and high compliance rates using the same dietary preparation method. ${ }^{22}$ Similar to their prior publication, the authors report an impressive indeterminate rate of $3 \%$ and only three patients excluded from the study due to diet nonadherence, again validating its effectiveness and high compliance rate.

With regards to scan interpretation, Patel and colleagues visually classified the myocardial FDG uptake patterns into: Negative for CS ("none" and "ring-like diffuse activity at base"), Positive for CS ("focal" uptake), and Indeterminate for CS (including "diffuse" and "focal on diffuse"). ${ }^{15}$ Other methods of image interpretation and pattern classification have been described, however until the recent publication of the 'Joint SNMMI-ASNC Expert Consensus Document on the Role of FDG PET/CT in Cardiac Sarcoid Detection and Therapy Monitoring,' none were formally established or validated with histological findings, ${ }^{23,24,25}$ thus setting up for inconsistent image interpretation. For example, while diffuse myocardial FDG uptake has been described as a normal variant by some investigators, ${ }^{12,26,27}$ Patel et al. categorize this finding as "indeterminate" ${ }^{15,22}$ There is also the mystery of FDG uptake in the lateral wall and ring-like diffuse activity at the heart base seen in some normal/healthy individuals. ${ }^{23,27}$ Moreover, Patel et al. make no reference to myocardial perfusion during image interpretation, which is considered important and essential by most practitioners. ${ }^{23}$ For example Blankstein and colleagues have put forth the following interpretation patterns; normal perfusion and metabolism, abnormal perfusion or metabolism and abnormal perfusion and metabolism. ${ }^{14}$ Okumura and colleagues have also shown the capability of FDG PET to detect early stage of cardiac sarcoidosis, in which fewer perfusion abnormalities and high inflammatory activity are noted. They proposed the following pathophysiologic classification, based on myocardial perfusion and FDG uptake patterns: "Normal" (normal perfusion/normal FDG uptake), "Early Stage" (no perfusion defect/increased FDG uptake), "Progressive Inflammatory Stage" (mild perfusion defect/ increased FDG uptake), "Peak Active Stage (moderate perfusion defect/increased FDG uptake), "Progressive Myocardial Impairment Stage" (severe perfusion defect/increased FDG uptake), and "Fibrosis Stage" (severe perfusion defect/ minimal or no FDG uptake). ${ }^{28,29}$ Further research correlating imaging findings with histology is needed. The recently published 'Joint SNMMI-ASNC Expert Consensus Document on the Role of ${ }^{18}$ F-FDG PET/CT in Cardiac Sarcoid Detection and Therapy Monitoring' is a comprehensive document that discusses the indications for cardiac PET/ CT for disease detection and management; provides recommendations on image acquisition, processing, and interpretation; and also reviews clinical scenarios in which PET/CT may help guide patient management. For image interpretation it recommends a normalized approach which involves simultaneous interpretation of perfusion and FDG images where the intensity of each image on perfusion and FDG is normalized to the maximum counts per pixel. ${ }^{25}$

Although not the focus of their study, Patel et al. make reference to the added benefit of treatment monitoring with FDG PET-CT by including example images of a case study of disease progression after 17-month steroid therapy that prompted change in treatment. ${ }^{15}$ FDG PET can be used to assess treatment response/ disease activity not only visually but also objectively by using different quantitative techniques. ${ }^{13,30,31}$ Ahmadian and colleagues nicely demonstrate that visual interpretation of FDG PET-CT images in cardiac sarcoidosis is less sensitive than quantitative measures (cardiac metabolic volume, cardiac metabolic activity and maximum standardized uptake value) for the assessment of response to immunosuppressive treatment. ${ }^{32}$ They also observed a rebound in quantitative FDG parameters after cessation of immunotherapy and acknowledge that reduction in the FDG signal has not been definitively proven to alter the disease progression or natural history of cardiac sarcoidosis. Although in a retrospective study, Yamagishi et al. describe seven patients diagnosed with cardiac sarcoidosis based on the Japanese Ministry of Health and Welfare guidelines that were treated with corticosteroids and underwent a cardiac ${ }^{13}$ NitrogenAmmonia $/{ }^{18}$ F-FDG PET a month later. While 
${ }^{13}$ Nitrogen-Ammonia myocardial perfusion defects exhibited no significant change, the FDG uptake was markedly diminished in size and intensity in five patients and disappeared completely in two patients. Furthermore, three of these patients demonstrated improvements and/or disappearance of conduction abnormalities or ventricular tachycardia. ${ }^{33}$ In addition, preliminary data by the University of Pennsylvania investigators has also shown that metabolic active volume and total lesion glycolysis are promising imaging biomarkers to evaluate disease progression. ${ }^{34}$ Prospective studies are needed to determine if quantitative changes in FDG uptake are associated with improved outcomes. Besides, an interval decrease in myocardial FDG uptake should be interpreted carefully and in combination with perfusion imaging, since it can represent not only treatment response but also postinflammatory scarring and fibrosis. ${ }^{29}$ The metabolic alterations related to prolonged corticosteroid therapy can also make performing and interpreting FDG images challenging. Elevations in serum glucose and insulin levels due to corticosteroid use may adversely affect FDG uptake by normal myocytes and reduce test specificity. ${ }^{29}$

In closing, while FDG PET is a powerful imaging technique for the diagnosis, prognosis and management of cardiac sarcoidosis there are several challenges and unanswered questions. Some of these include the lack of consensus on an optimal patient preparation method, the unclear association between disease outcome and treatment related visual and/or quantitative changes in FDG uptake, the incomplete suppression of physiologic myocardial FDG uptake in a small proportion of studies $(10 \%-15 \%)$ despite all interventions, ${ }^{35}$ prompting the need for an alternative/more specific inflammation imaging radiotracer etc. Clearly there is a lot more to learn. Until then, Patel and colleagues provide us with more evidence of the effectiveness of the 72-hour HFHPVLC diet preparation and make a strong case for whole body FDG PET-CT imaging over cardiac MRI to evaluate both cardiac and extracardiac burden of sarcoidosis.

\section{Disclosures}

There are no COI with this work.

\section{References}

1. Rybicki BA, Major M, Popovich J Jr, Maliarik MJ, Iannuzzi MC. Racial differences in sarcoidosis incidence: A 5-year study in a health maintenance organization. Am J Epidemiol. 1997; 145(3):234-41.
2. Gideon NM, Mannino DM. Sarcoidosis mortality in the United States 1979-1991: An analysis of multiple-cause mortality data. Am J Med. 1996;100(4):423-7.

3. Sekiguchi M, Hiroe M, Take M, Hirosawa K. Clinical and histopathological profile of sarcoidosis of the heart and acute idiopathic myocarditis. Concepts through a study employing endomyocardial biopsy. II. Myocarditis. Jpn Circ J. 1980;44(4): 264-73.

4. Sharma OP, Maheshwari A, Thaker K. Myocardial sarcoidosis. Chest. 1993;103(1):253-8.

5. Silverman KJ, Hutchins GM, Bulkley BH. Cardiac sarcoid: A clinicopathologic study of 84 unselected patients with systemic sarcoidosis. Circulation. 1978;58(6):1204-11.

6. Mehta D, Lubitz SA, Frankel Z, et al. Cardiac involvement in patients with sarcoidosis: Diagnostic and prognostic value of outpatient testing. Chest. 2008;133(6):1426-35.

7. Schatka I, Bengel FM. Advanced imaging of cardiac sarcoidosis. J Nucl Med. 2014;55(1):99-106.

8. Cooper LT, Baughman KL, Feldman AM, et al. The role of endomyocardial biopsy in the management of cardiovascular disease: A scientific statement from the American Heart Association, the American College of Cardiology, and the European Society of Cardiology Endorsed by the Heart Failure Society of America and the Heart Failure Association of the European Society of Cardiology. Eur Heart J. 2007;28(24):3076-93.

9. Buckley O, Doyle L, Padera R, et al. Cardiomyopathy of uncertain etiology: Complementary role of multimodality imaging with cardiac MRI and 18FDG PET. J Nucl Cardiol. 2010;17(2):328-32.

10. Patel MR, Cawley PJ, Heitner JF, et al. Detection of myocardial damage in patients with sarcoidosis. Circulation. 2009;120(20): 1969-77.

11. Youssef G, Leung E, Mylonas I, et al. The use of 18F-FDG PET in the diagnosis of cardiac sarcoidosis: A systematic review and metaanalysis including the Ontario experience. J Nucl Med. 2012;53(2):241-8.

12. Ohira H, Tsujino I, Ishimaru S, et al. Myocardial imaging with 18F-fluoro-2-deoxyglucose positron emission tomography and magnetic resonance imaging in sarcoidosis. Eur J Nucl Med Mol Imaging. 2008;35(5):933-41.

13. Mc Ardle BA, Birnie DH, Klein R, et al. Is there an association between clinical presentation and the location and extent of myocardial involvement of cardiac sarcoidosis as assessed by (1)(8)F- fluorodoexyglucose positron emission tomography? Circ Cardiovasc Imaging. 2013;6(5):617-26.

14. Blankstein R, Osborne M, Naya M, et al. Cardiac positron emission tomography enhances prognostic assessments of patients with suspected cardiac sarcoidosis. J Am College Cardiol. 2014;63(4): 329-36.

15. Patel DC, Gunasekaran SS, Goettl C, Sweiss NJ, Lu Y. FDG PETCT findings of extra-thoracic sarcoid are associated with cardiac sarcoid: A rationale for using FGD PET-CT for cardiac sarcoid evaluation. J Nucl Cardiol 2017.

16. Wielputz MO, Heussel CP, Herth FJ, Kauczor HU. Radiological diagnosis in lung disease: Factoring treatment options into the choice of diagnostic modality. Dtsch Arztebl Int. 2014;111(11): $181-7$.

17. Osborne MT, Hulten EA, Murthy VL, et al. Patient preparation for cardiac fluorine-18 fluorodeoxyglucose positron emission tomography imaging of inflammation. J Nucl Cardiol. 2017;24(1):86-99.

18. Harisankar CN, Mittal BR, Agrawal KL, Abrar ML, Bhattacharya A. Utility of high fat and low carbohydrate diet in suppressing myocardial FDG uptake. J Nucl Cardiol. 2011;18(5):926-36.

19. Soussan M, Brillet PY, Nunes H, et al. Clinical value of a high-fat and low-carbohydrate diet before FDG-PET/CT for evaluation of 
patients with suspected cardiac sarcoidosis. J Nucl Cardiol. 2013;20(1):120-7.

20. Manabe O, Yoshinaga K, Ohira H, et al. The effects of 18 -h fasting with low-carbohydrate diet preparation on suppressed physiological myocardial (18)F-fluorodeoxyglucose (FDG) uptake and possible minimal effects of unfractionated heparin use in patients with suspected cardiac involvement sarcoidosis. J Nucl Cardiol. 2016;23(2):244-52.

21. Scholtens AM, Verberne HJ, Budde RP, Lam MG. Additional heparin preadministration improves cardiac glucose metabolism suppression over low-carbohydrate diet alone in (1)(8)F-FDG PET imaging. J Nucl Med. 2016;57(4):568-73.

22. Lu Y, Grant C, Xie K, Sweiss NJ. Suppression of myocardial 18FFDG uptake through prolonged high-fat, high-protein, and verylow-carbohydrate diet before FDG-PET/CT for evaluation of patients with suspected cardiac sarcoidosis. Clin Nucl Med. 2017;42(2):88-94.

23. Dilsizian V, Bacharach SL, Beanlands RS, et al. ASNC imaging guidelines/SNMMI procedure standard for positron emission tomography (PET) nuclear cardiology procedures. J Nucl Cardiol. 2016;23(5):1187-226.

24. Ishida Y, Yoshinaga K, Miyagawa M, et al. Recommendations for (18)F-fluorodeoxyglucose positron emission tomography imaging for cardiac sarcoidosis: Japanese Society of Nuclear Cardiology recommendations. Ann Nucl Med. 2014;28(4):393-403.

25. Chareonthaitawee P, Beanlands RS, Chen W, et al. Joint SNMMIASNC expert consensus document on the role of ${ }^{18}$ F-FDG PET/ $\mathrm{CT}$ in cardiac sarcoid detection and therapy monitoring. J Nucl Cardiol. 2017;58(8):1341-53.

26. Ishimaru S, Tsujino I, Takei T, et al. Focal uptake on 18F-fluoro-2deoxyglucose positron emission tomography images indicates cardiac involvement of sarcoidosis. Eur Heart J. 2005;26(15): $1538-43$.
27. Nensa F, Tezgah E, Schweins K, et al. Evaluation of a low-carbohydrate diet-based preparation protocol without fasting for cardiac PET/MR imaging. J Nucl Cardiol. 2017;24(3):980-8.

28. Okumura W, Iwasaki T, Toyama $\mathrm{T}$, et al. Usefulness of fasting 18F-FDG PET in identification of cardiac sarcoidosis. J Nucl Med. 2004;45(12):1989-98.

29. Skali H, Schulman AR, Dorbala S. 18F-FDG PET/CT for the assessment of myocardial sarcoidosis. Curr Cardiol Rep. 2013;15(4):352.

30. Ahmadian A, Brogan A, Berman J, et al. Quantitative interpretation of FDG PET/CT with myocardial perfusion imaging increases diagnostic information in the evaluation of cardiac sarcoidosis. J Nucl Cardiol. 2014;21(5):925-39.

31. Tahara N, Tahara A, Nitta $\mathrm{Y}$, et al. Heterogeneous myocardial FDG uptake and the disease activity in cardiac sarcoidosis. JACC Cardiovasc Imaging. 2010;3(12):1219-28.

32. Ahmadian A, Pawar S, Govender P, Berman J, Ruberg FL, Miller EJ. The response of FDG uptake to immunosuppressive treatment on FDG PET/CT imaging for cardiac sarcoidosis. J Nucl Cardiol. 2017;24(2):413-24.

33. Yamagishi H, Shirai N, Takagi M, et al. Identification of cardiac sarcoidosis with (13)N-NH(3)/(18)F-FDG PET. J Nucl Med. 2003;44(7):1030-6.

34. Promteangtrong C, Salavati A, Alavi A. Assessment of treatment response of cardiac and extra-cardiac sarcoidosis by using metabolic and volumetric FDG PET/CT parameters. J Nucl Med. 2014;55(supplement 1):296.

35. Waller AH, Blankstein R. Quantifying myocardial inflammation using F18-fluorodeoxyglucose positron emission tomography in cardiac sarcoidosis. J Nucl Cardiol. 2014;21(5):940-3. 